\title{
Melioidosis Neck Abscess: An Opportunistic Infection in Diabetes Mellitus
}

\author{
How Kit Thong ${ }^{\mathrm{a}, \mathrm{b}}$, Puvan Arul ${ }^{\mathrm{a}}$
}

\begin{abstract}
Melioidosis is caused by the gram-negative environmental saprophyte, Burkholderia pseudomallei. This disease has caused significant morbidity and mortality to the diabetic population. Melioidosis can affect multiple systems; however, the reports on head and neck regions are relatively uncommon. The disease is endemic particularly in the Southeast Asia and northern Australia. We here report a case of neck abscess caused by B. pseudomallei, which is a rare but an important differential diagnosis of neck abscess.
\end{abstract}

Keywords: Melioidosis; Melioidosis neck abscess; Neck abscess

\section{Introduction}

Melioidosis infection usually follows percutaneous inoculation or inhalation of the causative bacterium, Burkholderia pseudomallei, which is present in soil and surface water in the endemic region. A recent study was done in the state of Johor which is the origin of our patient, reporting that vast majority of the cases have identified risk factors, the most significant of which are diabetes mellitus $(75 \%)$ and chronic renal disease $(12.5 \%)$ [1]. Diagnosis of the disease is by culture of B. pseudomallei from blood, sputum, throat swab or other samples.

\section{Case Report}

A 55-year-old Malay lady with underlying poorly controlled diabetes mellitus was hospitalized with a 2-week history of neck swelling, progressively increasing in size. She also complained of high grade fever prior to the development of the neck swell-

Manuscript accepted for publication July 06, 2016

aDepartment of Otorhinolaryngology-Head \& Neck Surgery, Hospital Sultan Ismail, Johor Bahru, Malaysia

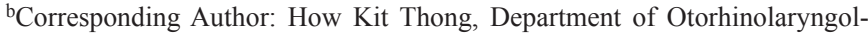
ogy-Head \& Neck Surgery, Hospital Sultan Ismail, Johor Bahru, Malaysia. Email: t.howkit@gmail.com

doi: http://dx.doi.org/10.14740/jmc2580w ing. On further history noted, patient had recently visited a leisure park in Kota Tinggi, a district located in the state Johor, an area where melioidosis is known to be endemic. On admission, she was subfebrile; however, her other vital signs were stable. Physical examination revealed a huge erythematous fluctuant swelling at the left submandibular region measuring $8 \times 5 \mathrm{~cm}$, and her oral examination showed that she had very poor oral hygiene with multiple cavities. Laboratory tests revealed a white blood cell count of 13,500 cells/ $\mu \mathrm{L}, \mathrm{C}$-reactive protein level of $147 \mathrm{mg} / \mathrm{dL}$, ESR $66 \mathrm{~mm} / \mathrm{h}$, fasting blood sugar of $10.0 \mathrm{mmol} / \mathrm{L}$ and HBA1c of $11.7 \%$. Computed tomography (CT) of neck showed an abscess collection at right side of neck measuring 4.8 $\times 6.8 \times 8.9 \mathrm{~cm}$. Superiorly the collection extended to pterygoid plate level; inferiorly it extended until cricoid cartilage level with multiple lobulated lesion with central hypodensity, medial to the collection suggestive of necrotic node (Fig. 1).

Clinically our diagnosis was right neck abscess secondary to dental origin and we proceeded with neck exploration drainage and dental clearance under general anesthesia. The patient was placed under observation with postoperative administration of sulperazone. Postoperatively, the wound remained unhealthy with a lot of slough tissue. On postoperative day 4 , we noted a new lesion on the left submandibular region measuring $2 \times 2 \mathrm{~cm}$. An ultrasound was performed and there was a new left submandibular lesion measuring $2 \times 0.9$ $\mathrm{cm}$ suggestive of suppurative lymph node. The culture of the abscess fluid which was drained intraoperatively was positive for $B$. pseudomallei sensitive to ceftazidime, meropenen and augmentin. The antibiotic therapy was changed to ceftazidime based on the sensitivities of the cultured organisms. An ultrasound abdomen was also performed; however, no other foci of infections were found. A repeated swab culture was taken from the wound on postoperative day 4 which was also positive for B. pseudomallei. Wound management included daily wound debridement with 6 hourly dressing with diluted povidone. After commencement of the intravenous ceftazidime and the frequent dressing, the wound improved with appearance of granulation tissue, and the left submandibular suppurative lymphadenitis also shrunk with near resolution. The patient was discharged from the ward after completing 2 weeks of intravenous ceftazidime. Eradication therapy with oral doxycycline $200 \mathrm{mg}$ daily was started. She was followed up at our outpatient clinic 2 weeks later, and there was no recurrence of abscesses during the follow-up visit. We can only speculate about this patient's potential sources of infection, which 


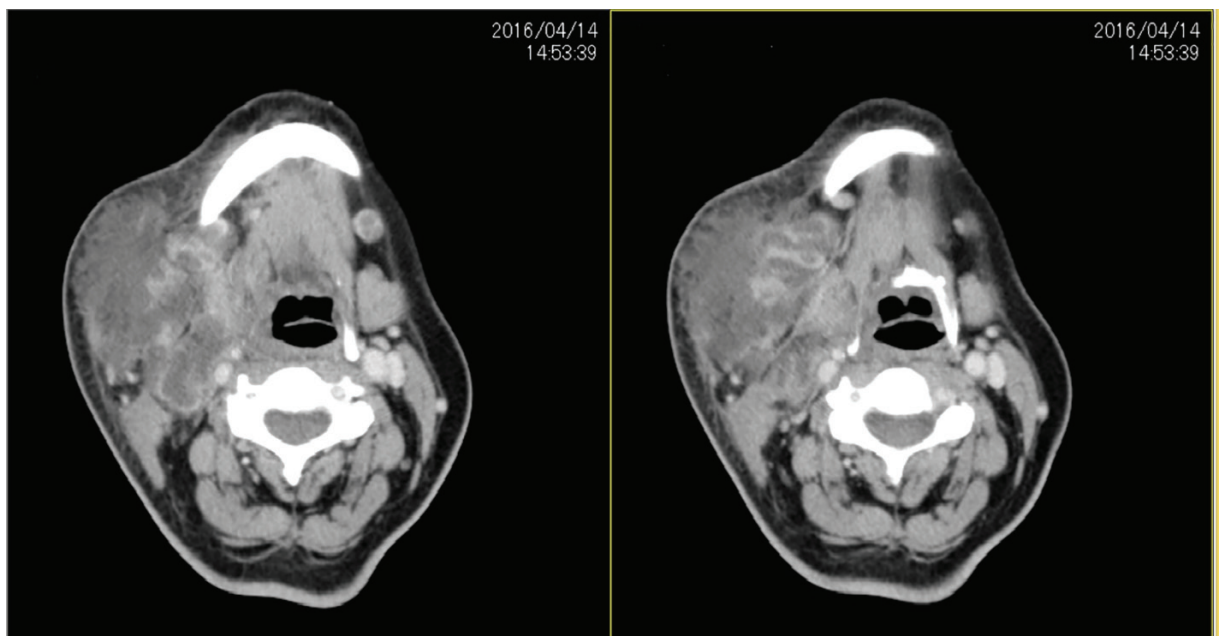

Figure 1. CT scan showing hypodense fluid collection with enhanced wall in the left submandibular space.

included minor abrasions from walking barefoot around the leisure park and inhalation or swallowing of the causative organisms during water activities.

\section{Discussion}

Melioidosis is caused by the gram-negative saprophyte $B$. pseudomallei, and is a disease of public health importance in Southeast Asia. Melioidosis predominantly affects people whom had contact with soil and water. The commonest routes of $B$. pseudomallei infection are thought to be inoculation, inhalation and ingestion [2]. The disease can affect a spectrum of organs, and its clinical manifestations can range from localized abscesses to acute pneumonia, and fulminant septic melioidosis [3-5]. Once infected, it may remain dormant and become active after months, years or decades when host is immunocompromised by drugs (steroids) or systemic diseases (diabetes mellitus and chronic renal failure) or social deprivation (alcoholism, drug abuse, and occupational exposure) [6]. Our patient is immunocompromised as she has a very poorly controlled diabetic status (HBA1c: $11.7 \%$ ) which predisposed her to the disease. Although melioidosis can affect any part of the body, it is somewhat surprising that there are only a handful of reports involving melioidosis in ENT [7]. Head and neck infections normally arise mainly from Klebsella pneumoniae, Streptococcus, Staphylococcus, Haemophilus, or other anaerobic species, and infection by $B$. pseudomallei, is rare. A retrospective analysis of 158 cases of neck abscesses has been done by Lee et al showing that $K$. pneumoniae $(13.7 \%$ ) was the most common pathogen among the positive cultures, followed by Streptococcus viridans (12.3\%), methicillin-sensitive Streptococcus aureus (MSSA) (11.0\%), and $\alpha$-hemolytic Streptococcus $(8.2 \%)$. Mixed infection reached 9.6\% [8].

In our case, a diagnosis of neck abscess secondary to dental origin was made initially. When the culture had grown $B$. pseudomallei, the patient was immediately put on intravenous ceftazidime and it prevented the formation of a second abscess in the left submandibular region, the drainage of the abscess and a sensitive antibiotic is of prime importance in the management of melioidosis for the fear of progression to a fulminant course.

Ceftazidime is the drug of choice in systemic melioidosis. Ceftazidime (120 mg/kg/day) has shown to reduce the mortality significantly in severe melioidosis [9].

Ceftazidime then became the drug of choice for initial intensive therapy for melioidosis. Another study from Thailand showed similar results when ceftazidime was used in combination with cotrimoxazole for initial intensive therapy; they recommend ceftazidime and cotrimoxazole as the drugs of choice for treatment of severe melioidosis, especially in those patients with disseminated septicemia [10]. A randomized trial by Chetchotisakd et al found reduction in mortality on using a combination of ceftazidime and cotrimoxazole [11]. Following initial intensive therapy, using ceftazidime, imipenem or meropenem, possibly in combination with cotrimoxazole, subsequent eradication therapy is compulsory for preventing relapse of melioidosis. Our patient was prescribed oral doxycycline $100 \mathrm{mg}$ twice daily for a planned duration of 4 months. Conventional treatment of melioidosis abscess includes surgical drainage of abscess and appropriate intravenous antibiotics for a minimum of 2 weeks [7].

\section{Conclusion}

We described a case of neck abscess caused by B. pseudomallei in a patient with poorly controlled diabetes mellitus, who was treated with surgical drainage and antibiotics. The moral of the story is neck abscess by B. pseudomalle $i$ which is a rare disease, but an important differential diagnosis of neck abscess in patients with risk factors.

\section{References}

1. Pagalavan L. Melioidosis: the Johor Bahru experience. Med J Malaysia. 2005;60(5):599-605. 
2. Cheng AC, Currie BJ. Melioidosis: epidemiology, pathophysiology, and management. Clin Microbiol Rev. 2005;18(2):383-416.

3. Raja NS. Localized melioidosis. J Pak Med Assoc. 2003;53(8):373-374.

4. Koszyca B, Currie BJ, Blumbergs PC. The neuropathology of melioidosis: two cases and a review of the literature. Clin Neuropathol. 2004;23(5):195-203.

5. Schwarzmaier A, Riezinger-Geppert F, Schober G, Karnik R, Valentin A. Fulminant septic melioidosis after a vacation in Thailand. Wien Klin Wochenschr. 2000;112(20):892-895.

6. Currie B, Howard D, Nguyen VT, Withnall K, Merianos A. The 1990-1991 outbreak of melioidosis in the Northern Territory of Australia: clinical aspects. Southeast Asian J Trop Med Public Health. 1993;24(3):436-443.

7. Lim WK, Gurdeep GS, Norain K. Melioidosis of the head and neck. Med J Malaysia. 2001;56(4):471-477.
8. Lee JK, Kim HD, Lim SC. Predisposing factors of complicated deep neck infection: an analysis of 158 cases. Yonsei Med J. 2007;48(1):55-62.

9. Suputtamongkol Y, Chaowagul W. Management and therapy of melioidosis. First international symposium on Melioidosis in Kuala Lumpur Malaysia. 1994;7-8.

10. Sookpranee M, Boonma P, Susaengrat W, Bhuripanyo K, Punyagupta S. Multicenter prospective randomized trial comparing ceftazidime plus co-trimoxazole with chloramphenicol plus doxycycline and co-trimoxazole for treatment of severe melioidosis. Antimicrob Agents Chemother. 1992;36(1):158-162.

11. Chetchotisakd P, Porramatikul S, Mootsikapun P, Anunnatsiri S, Thinkhamrop B. Randomized, double-blind, controlled study of cefoperazone-sulbactam plus cotrimoxazole versus ceftazidime plus cotrimoxazole for the treatment of severe melioidosis. Clin Infect Dis. 2001;33(1):29-34. 